\title{
Photocatalytic water splitting with noble-metal free cocatalysts for a comprehensive study of two nonidentical photoreactors designs
}

\author{
Jean César Marinozi Vicentini ${ }^{1}$ (i) | Rafael Manieri $^{1}$ | Gimerson Weigert Subtil ${ }^{1}{ }^{\infty}$ | \\ Fernanda de Oliveira Tavares ${ }^{1}$ (1) | Daiane Marques de Oliveira ${ }^{1}$ | \\ Patrícia Hissae Yassue-Cordeiro $^{2}$ | Heveline Enzweiler ${ }^{3}$ | Marcelino Luiz Gimenes ${ }^{1}$ | \\ Maria do Carmo Martins Alves ${ }^{4}$ | Jonder Morais ${ }^{5}$ () | | \\ Mara Heloisa Neves Olsen Scaliante ${ }^{1}$ | Marcos de Souza ${ }^{1}$
}

${ }^{1}$ Chemical Engineering Department, State

University of Maringá, Maringá, Brazil

${ }^{2}$ Chemical Engineering Department, Federal University of Technology - Paraná, Londrina, Brazil

${ }^{3}$ Chemical Engineering and Food Engineering Department, State University of Santa Catarina, Pinhalzinho, Brazil

${ }^{4}$ Institute of Chemistry, Federal University of Rio Grande do Sul, Porto Alegre, Brazil

${ }^{5}$ Institute of Physics, Federal University of Rio Grande do Sul, Porto Alegre, Brazil

\section{Correspondence}

Jean César Marinozi Vicentini, Chemical Engineering Department, State University of Maringá, Av. Colombo, 5790, Maringá, Paraná, CEP 87020900, Brazil.

Email: jcmarinozi@gmail.com

Funding information

Conselho Nacional de Desenvolvimento Científico e Tecnológico; Coordenação de Aperfeiçoamento de Pessoal de Nível Superior

\begin{abstract}
Here, the authors (i) discuss the most prominent co-catalyst for $\mathrm{H}_{2}$ generation structured in the form of $\mathrm{Me}-\mathrm{TiO}_{2} / \mathrm{MCM}-41$ (Me: $\mathrm{Ag}, \mathrm{Co}, \mathrm{Cu}, \mathrm{Ni}$ ) based on structural, electronic, textural, morphological and optical characterization techniques, such as XRD, wide and small angle, XPS, Fourier-transform infrared spectroscopy, scanning electron microscopy, B.E.T., textural analysis, photoacoustic spectroscopy and photoluminescence spectroscopy; and (ii) evaluate the difference in hydrogen production in two distinct geometric reactors based on a theoretical study of light distribution inside the reactors supported by the experimental quantum yield calculation. As a result, copper-doped photocatalyst generated higher hydrogen amount compared to the others. The high photocatalyst performance was due to the greater lamp spectrum absorption, marked by the low bandgap value, and high photoactivity justified by the low rate of electronic recombination. The hydrogen generation in the quartz reactor was seven times higher than the annular one, and when at maximum light power, it is comparable to the most sophisticated reaction systems found in literature. The larger light exposure area per unit volume of the quartz reactor compared to the annular one is the reason why it obtained better results due to the lower emitted photon blockade, with a $1.81 \%$ apparent quantum yield.
\end{abstract}

\section{KEYWORDS}

hydrogen production, photocatalysis

\section{1 | INTRODUCTION}

According to the National Oceanic and Atmospheric Administration, in July 2019, the historical average global temperature record was considered to be the hottest month ever observed, reaching a mark of $16.7^{\circ} \mathrm{C}\left(62.11^{\circ} \mathrm{F}\right)$. This is due, in fact, to the $\mathrm{CO}_{2}$ concentration increase in the atmosphere, which reached the historical mark of
$411.7 \mathrm{ppm}$ in the same period. With this aggravation, the development of clean and sustainable technologies has become a recurring theme on global meetings, and one of the most relevant themes in environmental science is the mission of recovering our natural biodiversity and guaranteeing quality of life.

Hydrogen is a clean source of energy and it can be achieved from different renewable sources, such as catalytic reforming of acids and 
alcohols, ${ }^{1-4}$ and also from catalytic hydrolysis of sodium borohydride ${ }^{5}$ and water. ${ }^{6-17}$ Solar energy and water are potentially the most abundant renewable energy sources available on the planet, ${ }^{14,18}$ so their combination creates an environmentally friendly reaction mechanism for the hydrogen production. Hydrogen production by catalytic photolysis of water is considered one of the most promising technologies for producing this alternative energy source. The main water splitting mechanisms for hydrogen production include photon absorption by the active catalyst phase, electronic excitation from the valence band to the conduction band, charges separation, and subsequent catalytic reactions at the catalyst surface. ${ }^{18}$

The low $\mathrm{H}_{2}$ production efficiency achieved with titanium oxides catalysts is due in particular to reasons such as: fast electronic recombination (which leads to the unproductive energy dissipation by the photocatalyst, causing its subsequent deactivation), low stability, slow kinetics inhibiting industrial applications, rapid reverse reaction (leading to rapid products recombination back in water), and visible light inefficient absorption. ${ }^{6,10,19,20}$ In this context, the use of cocatalysts, as photosensitizers, aims to accelerate charge separation and electron sequestration in order to reduce the electron recombination time, increase the amount of available active sites and introduce intrinsic defects, promoting the narrowing of the photocatalyst bandgap and changes in its electronic and optical properties. ${ }^{6-8,11,18-20}$

More recently, it has been published that transition metals such as copper, cobalt, and nickel may present a higher reaction yield of hydrogen production than noble metals such as silver, palladium, and platinum. ${ }^{13,18,21}$ This higher yield is justified by the large capacity of these metals to sequester the photoexcited electrons allowing them to remain longer time for reaction, since when combined with $\mathrm{TiO}_{2}$ they reduce the bandgap energy. ${ }^{22}$ These metals are less expensive and offer greater advantage when compared to noble metals. Using $2 \% \mathrm{Co} / \mathrm{TiO}_{2}$, for example, $77.8 \%$ of the total $\mathrm{H}_{2}$ obtained using $1 \%$ $\mathrm{Pt} / \mathrm{TiO}_{2}$ catalyst was achieved ${ }^{8}$; similarly when using $8 \% \mathrm{Cu} / \mathrm{TiO}_{2}$ obtained a production $20 \mathrm{mmol}\left(\mathrm{H}_{2}\right) \mathrm{h}^{-1} \mathrm{~g}^{-1,13}$ which is greater than using $2 \% \mathrm{Au} / \mathrm{TiO}_{2}\left(2.7 \mathrm{mmol} \mathrm{g}{ }^{-1} \mathrm{~h}^{-1}\right)$ and $1 \% \mathrm{Pd} / \mathrm{TiO}_{2}(2.1 \mathrm{mmol} \mathrm{g}$ $\left.{ }^{1} \mathrm{~h}^{-1}\right)^{23}$ or $0.96 \mathrm{gL}^{-1}$ of $0.6 \% \mathrm{Pt} / \mathrm{TiO}_{2}(6000 \mu \mathrm{mol}) .{ }^{24}$ Another example is the $\mathrm{Ni} / \mathrm{TiO}_{2}$ catalyst, which presents a yield compared to noble metals, of approximately $26 \mathrm{mmol}\left(\mathrm{H}_{2}\right) \mathrm{g}^{-1} \mathrm{~h}^{-1,25}$ greater than $910 \mu \mathrm{mol}\left(\mathrm{H}_{2}\right) \mathrm{g}^{-1} \mathrm{~h}^{-1}$ achieved for $\mathrm{Ag} / \mathrm{TiO}_{2}{ }^{26}$

The impregnation on supports, besides giving a greater specific area, stability, and recyclability to the catalyst, can also act in synergy with cocatalysts, acting in the reagents adsorption, and in the transport and sequestration of charges. ${ }^{1,7,8,10,21,27,28}$ The MCM-41 (Mobil Composition of Matter, number 41) belongs to the ordered material family M41S and the symmetry group p6mm. ${ }^{29-31}$ The MCM-41 is formed by a hexagonal mesoporous arrangement and has a parallel porous system and linear one-dimensional defined channels. ${ }^{1,30}$ The MCM-41 mesoporous arrangement is achieved by calcination of the material obtained by polycondensation of the silanol groups, formed after the $\mathrm{pH}$ reduction, of the alkaline anions silicate solution and polymeric surfactant cations. ${ }^{32}$ It is a typical low acidity mesoporous molecular sieve, which justifies the fact that it is subjected to surface modification by metallic impregnations to increase the acidity for applications requiring such surface characteristics. ${ }^{33}$ Active phase and supports may have a cooperative effect on the reactions employed, however, there is a concentration of the active phase and a concentration of the impregnated catalyst that is considered ideal for application. Ideal catalyst concentrations are an important factor for photocatalytic reactions, as catalyst insufficiency and excess can compromise not only reaction efficiency but the material and operating costs. $6,8,10,19,20,27,31$

The catalyst composition and the experimental reaction conditions such as temperature, pressure, catalyst concentration, active phase, or sacrifice agents are factors that can fluctuate the photocatalytic reaction yield. Other factors such as the geometry, composition of the reactor's inner walls, type, and arrangement of the radiation source and catalytic solutions' optical properties are also very important since they are variables that influence light distribution in photocatalytic reactors. In other words, it influences how the catalyst will be dispersed in the reaction medium, to what extent the irradiation will be absorbed by the catalyst surface, and how the photons will be harnessed for the electron/hole pairs generations. ${ }^{18,34,35}$

Light distribution in the reactor is the basis of design parameters for photocatalytic reactors, such as local volumetric rate of energy absorption (LVREA) profiles, which refer to the amount of radiation absorbed by the photocatalyst particle per unit of time and volume. LVREA profiles are widely used in the literature to determine the optimal photocatalyst concentration to avoid dark zone formation since it is clear from their direct relationship that a possible increase in catalyst concentration can affect light penetrability in the reaction medium. ${ }^{19,34-39}$ According to the authors, the LVREA profile when evaluated on the photoreactors' radial axis indicates an exponentially decrease as it approaches the inner wall of the reactor, that is, when most of the photons are being absorbed or scattered closer to the lamp. ${ }^{34-36}$

There are few studies comparing the effect of the catalyst on hydrogen production under the same operating and experimental conditions in reactors of different geometries. Koci and coauthors investigated the effect that the reactor geometry exerts on catalytic $\mathrm{CO}_{2}$ photoreduction in reaction yield. They concluded that the best reaction performance is obtained in batch reactors, with the radiation source tangent to the liquid phase and unimpeded mechanical agitation, favoring these reactors over the annular ones. The fluid movement in annular reactors is not good, causing a non-uniform catalyst concentration throughout the volume and consequently a less effective light absorption by the photocatalyst. ${ }^{40}$

This work aims to further extend the literature regarding a detailed and well-referenced comparative experimental study between two distinct geometry reactors in the $\mathrm{H}_{2}$ production from water splitting under the same experimental and operational conditions of temperature, pressure, catalyst, sacrificial agent concentration, catalyst compositions, and light source. And also seek to evaluate by the use of several characterization techniques, which of the metals $(\mathrm{Ag}, \mathrm{Ni}, \mathrm{Cu}$, and $\mathrm{Co}$ ) is the best doping agent on binary catalyst Ti/MCM-41 for hydrogen generation. 


\section{2 | MATERIALS AND METHODS}

Titanium (IV) isopropoxide (Ti[OCH $\left.\left[\mathrm{CH}_{3}\right]_{2}\right]_{4}, 97 \%$, Sigma-Aldrich, CAS $\mathrm{N}^{\circ}$ 546-68-9), ammonium hydroxide $\left(\mathrm{NH}_{4} \mathrm{OH}, 28-30 \%\right.$, Anidrol, CAS $\mathrm{N}^{\circ}$ 1336-21-6), hexadecyltrimethylammonium bromide (CTAB, $\mathrm{CH}_{3}\left(\mathrm{CH}_{2}\right)_{15} \mathrm{~N}(\mathrm{Br})\left(\mathrm{CH}_{3}\right)_{3}$, min. 99\%, Sigma-Aldrich, CAS N $\mathrm{N}^{\circ}$ 57-09-0), tetraethyl orthosilicate reagent grade (TEOS, $\mathrm{Si}\left[\mathrm{OC}_{2} \mathrm{H}_{5}\right]_{4}, 98 \%$, SigmaAldrich, CAS $\mathrm{N}^{\circ}$ 78-10-4), copper (II) nitrate $\left(\mathrm{Cu}\left[\mathrm{NO}_{3}\right]_{2} \cdot 3 \mathrm{H}_{2} \mathrm{O}\right.$, 99-104\%, Sigma Aldrich, CAS N ${ }^{\circ}$ : 13778-31-9), cobalt (II) nitrate (Co $\left[\mathrm{NO}_{3}\right]_{2} \cdot 6 \mathrm{H}_{2} \mathrm{O}$, min. 98\%, Dinâmica, CAS $\mathrm{N}^{\circ}:$ 10026-22-9), silver nitrate $\left(\mathrm{AgNO}_{3}\right.$, min. 99\%, Sigma-Aldrich, CAS No. 7761-88-8), nickel (II) nitrate $\left(\mathrm{Ni}^{2}\left[\mathrm{NO}_{3}\right]_{2} .6 \mathrm{H}_{2} \mathrm{O}, 98-102 \%\right.$, Dinâmica, CAS $\mathrm{N}^{\circ}$ : 13478-00-7), isopropyl alcohol $\left(\mathrm{C}_{3} \mathrm{H}_{7} \mathrm{OH}\right.$, min.99.5\%, Synth, CAS $\mathrm{N}^{\circ}$ 67-63-0), ethyl alcohol $\left(\mathrm{CH}_{3} \mathrm{CH}_{2} \mathrm{OH}\right.$, min. 99.5\%, Synth, CAS $\mathrm{N}^{\circ}$ 64-17-5), $\mathrm{N}_{2}$ gas (purity $>99.997 \%, 4.8$, Messer group).

\section{3 | CHARACTERIZATION}

The photocatalysts synthesis was described in the Supplementary Material. The prepared photocatalysts were characterized by wide ( $40 \mathrm{kV}$, current of $30 \mathrm{~mA}$, range of $10-80^{\circ}$, velocity of $2^{\circ} / \mathrm{min}$, step of $0.02^{\circ}$ ) and small (range of $0^{\circ}-8^{\circ}$ ) X-ray diffraction (Shimadzu LabX XRD 6000), photoacoustic spectroscopy (PAS, detailed technical information in the Supplementary Material), scanning electron microscopy (Microscopy Quanta 250, Oxford Instruments, $20 \mathrm{kV}$, spot 3.5, $45 \mathrm{~s}$ of gold sputtering) and energy-dispersive X-Ray spectroscopy (EDS, Microscopy Quanta 250, Oxford Instruments, $20 \mathrm{kV}$, spot 4, $45 \mathrm{~s}$ of gold sputtering), Fourier-transform infrared spectroscopy (FTIR, Varian, $\mathrm{KBr}$ pattern, 16 scans, $4 \mathrm{~cm}^{-1}$ resolution, $5 \mathrm{kHz}, 4000-400 \mathrm{~cm}^{-1}$ ), textural analysis (detailed technical information in the Supplementary Material, Micromeritics ASAP 2020), photoluminescence spectroscopy (PL, Perkin Elmer LS45, excitation at $\lambda=250 \mathrm{~nm}$, optical filter type high pass at $\lambda=320 \mathrm{~nm}$, slot width of $10 \mathrm{~nm}$ and velocity scan of $100 \mathrm{~nm} / \mathrm{s}$ ) and X-ray photoelectron spectroscopy (XPS, detailed technical information in the Supplementary Material).

\section{4 | PHOTOCATALYTIC REACTION}

The photocatalytic water splitting was conducted in two distinct reactors.

1. Annular reactor designed by Enzweiler and coauthors ${ }^{6}$ (Figure S1 in the Supplementary Material). Unlike Enzweiler and coauthors, here the authors used an $18 \mathrm{~W}$ UV-C lamp (Model HNS L by Osram) as a radiation source positioned in the reactor axis. The reactor had $10 \mathrm{~cm}$ of inner diameter, and the lamp was protected by a $4 \mathrm{~cm}$ diameter quartz tube. The liquid phase corresponded to $600 \mathrm{ml}$ and the light exposure area in contact with the solution was $101 \mathrm{~cm}^{2}$, corresponding to an area/volume ratio (A/V) of $0.16 \mathrm{~cm}^{2} \mathrm{ml}^{-1.6}$

2. Quartz reactor (Figure S2 in the Supplementary Material). The reaction system was designed for this purpose and comprised a system that could combine up to three $18 \mathrm{~W}$ UV-C lamps (Model HNS L by Osram) arranged in an anodized aluminum refractory arc external to the reactor. The liquid phase corresponded to $30 \mathrm{ml}$ and established a thin layer of $1.3 \mathrm{~cm}$ corresponding to an illuminated area exposition of approximately $96 \mathrm{~cm}^{2}$ and an $A / V$ of $3.2 \mathrm{~cm}^{2} \mathrm{ml}^{-1}$.

Although the quartz reactor had a smaller volume, it had an $A / V$ 20 times greater than the annular reactor, without higher energy costs, considering the quartz reactor has only two lamps more than the annular one. And since the reaction is photocatalytic, greater light exposure tends to offer greater efficiency for the catalysts in the reaction.

In both systems, the catalysts were dispersed in an aqueous ethanol solution (10\%), corresponding to an excess of sacrificial agent, ${ }^{10}$ at a catalyst concentration of $1 \mathrm{~g} / \mathrm{L}(\mathrm{Dp}<0.35 \mathrm{~mm})$. The catalysts compositions were $1 \% \mathrm{Me} / 20 \% \mathrm{TiO}_{2} / \mathrm{MCM}-41(\mathrm{Me}=\mathrm{Ag}, \mathrm{Co}, \mathrm{Cu}, \mathrm{Ni})$ and their syntheses are placed at Supplementary Material. The reaction medium was previously purged with nitrogen for $20 \mathrm{~min}$ to remove oxygen and kept at $25^{\circ} \mathrm{C}$ during the $4 \mathrm{~h}$ irradiation period. Hydrogen production was measured at regular intervals of $1 \mathrm{~h}$ using a Trace GC gas chromatography and Carboxen ${ }^{\mathrm{TM}} 1000$ capillary column with thermal conductivity detector.

\section{5 | RESULTS AND DISCUSSION}

\section{1 | Materials characterization}

Low angle X-ray diffraction patterns of pure MCM-41 and titanium dioxide catalyst were obtained to evaluate the matrix mesoporous structure (Figure 1). The synthesis of MCM-41 has been successfully

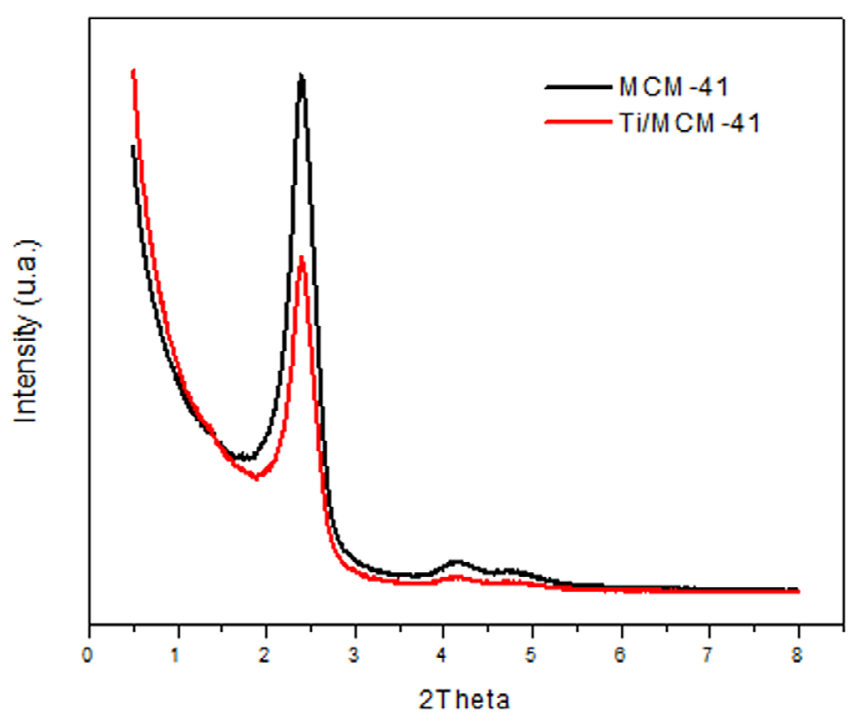

FIG URE 1 Small angle XRD patterns from MCM-41 and $\mathrm{Ti} / \mathrm{MCM} / 41$ catalyst [Color figure can be viewed at wileyonlinelibrary.com] 
performed since the hexagonal ordering of the mesoporous structure can be confirmed by three characteristic signals comprised in the bands at $2 \theta=2.4^{\circ}, 4.1^{\circ}-4.2^{\circ}$, and $4.9^{\circ}-5.1^{\circ}$, which are indexed to plans (100), (110), and (200). ${ }^{1-4,21,28-31,33,41-44}$

According to MCM-41 diffractograms collected before and after $\mathrm{TiO}_{2}$ impregnation, no peak displacements were observed, indicating that the impregnation process did not promote any structural modification in the mesoporous support, ${ }^{29,31,45-47}$ with only a decrease in relative crystallinity in the diffraction signal intensity of the plane (100). One may notice that the decrease in relative crystallinity, accompanied to the larger broadening of this plane, confirms that $\mathrm{TiO}_{2}$ incorporation contributes to the formation of large MCM-41 crystal particles during the catalyst synthesis. ${ }^{1,2,4,8,21,27}$ Since larger particles were obtained, a leak of porosity might also occur.

According to small-angle XRD patterns (Figure 2), for metallic photocatalysts, it was possible to observe that there were peaks that disappear at low-angle and it is suggested, therefore, that there was an insertion of these metals in the structure of MCM-41, except for $\mathrm{Ag}-\mathrm{Ti} / \mathrm{MCM}-41$. For the catalyst impregnated with silver, the MCM41 plane was observed (Figure 2), so it suggests that the silver preferably remained on the molecular sieve surface. Wide-angle XRD

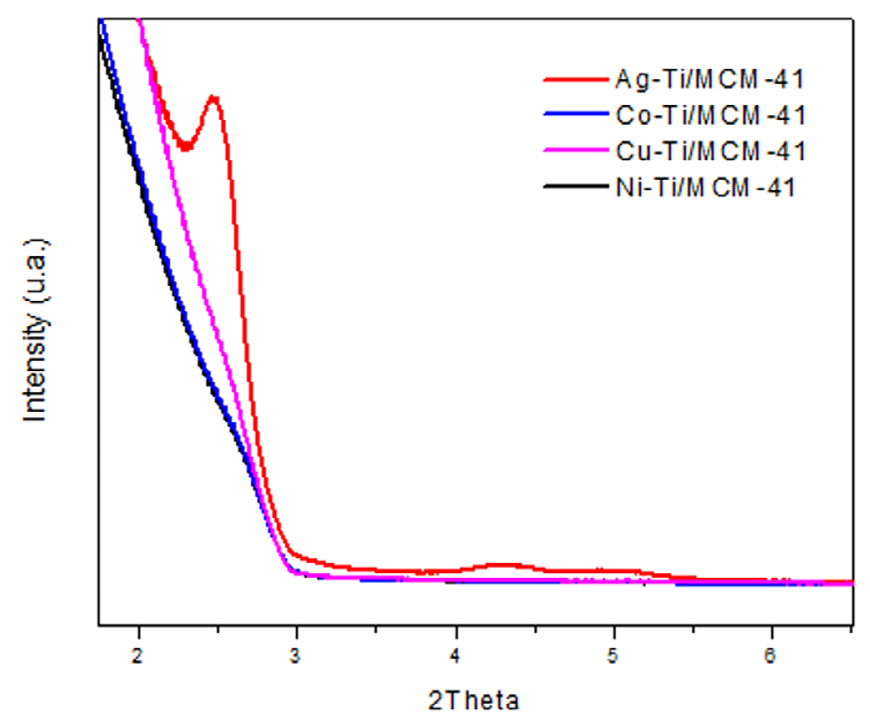

FIGURE 2 Small angle XRD patterns from $\mathrm{Ag}, \mathrm{Co}, \mathrm{Cu}$, and $\mathrm{Ni}$ cocatalysts decorated on Ti/MCM-41 catalyst [Color figure can be viewed at wileyonlinelibrary.com] patterns were shown in Figure S3 in the Supplementary Material indicating the anatase-phase of $\mathrm{TiO}_{2}$ and others observations.

The MCM-41 specific area is $1275 \mathrm{~m}^{2} / \mathrm{g}$, close to the value found by Cakirylmaz et al. and Nejat et al. ${ }^{3,12}$ According to Table 1 of textural analysis it was possible to see a decrease in the catalyst specific area due to the metal impregnation method. ${ }^{1,4,10,29}$ Unlike the specific area, pore diameter increased after metal impregnation, caused by clogging of smaller pores and subsequently increasing the mean particle diameter. ${ }^{27,31}$ The higher pore volume of the Ag-Ti/MCM-41 catalyst compared to the others matches with the discussion about low-angle XRD (shown in Figure 2), which was the only photocatalyst that indicated the peak of MCM-41, due to the metal external surface recovering, that may contribute to additional porosity formation, as observed by textural analysis, but not observed from the other metals impregnation. In general, a good catalyst distribution on molecular sieve surface could be concluded from EDS spectra at Figure S6 presented in the Supplementary Material.

Surface properties were analyzed by FTIR (Figure S7a in the Supplementary Material) to identify the functional groups present in all prepared samples. The absorption bands in the range of $3200-3600 \mathrm{~cm}^{-1}$ and $1700 \mathrm{~cm}^{-1}$ present in all samples are attributed to the hydroxyl group stretching mode (physiosorbed and coordinated water, respectively) and the possible presence of moisture in the samples, which may be due to the $\mathrm{KBr}$ humidity. ${ }^{1,2,29-31,42,43,48}$ Absorption bands in the range $1030-1080 \mathrm{~cm}^{-1}$ are asymmetric stretching vibrations of Si-O-Si bonds and bands between $780-805 \mathrm{~cm}^{-1}$ are due to symmetrical stretching vibration of Si-O-Si bonds. ${ }^{1,2,21,28,29,31,42,43,48}$ Bands in the $450-470 \mathrm{~cm}^{-1}$ range are due to $\mathrm{Si}-\mathrm{O}$ bending vibration. $^{21,29,43}$

Based on the FTIR spectra, the widening of the vibration band in the corresponding $962-970 \mathrm{~cm}^{-1}$ range observed in the spectrum after metal loading may be due to $\mathrm{Si}-\mathrm{O}-\mathrm{M}$ vibration $\mathrm{M}=\mathrm{Ti}, \mathrm{Cu}, \mathrm{Co}$, $\mathrm{Ag}$, and $\mathrm{Ni}$ ). ${ }^{21,29,42}$ It was also observed a red-shift from $805 \mathrm{~cm}^{-1}$ to $800,796,790,798$ e $796 \mathrm{~cm}^{-1}$, referring to the asymmetric stretch vibrations $\mathrm{Ti}-\mathrm{O}-\mathrm{Ti}$, Ti-O-Ni, Ti-O-Cu, Ti-O-Co, and Ti-O-Ag, respectively. ${ }^{20,49}$ Blue-shifts were also identified from $466 \mathrm{~cm}^{-1}$ to $453,450,453,450,451 \mathrm{~cm}^{-1}$ and they would describe as stretching mode for $\mathrm{Ti}-\mathrm{O}, \mathrm{Ni}-\mathrm{O}, \mathrm{Cu}-\mathrm{O}, \mathrm{Co}-\mathrm{O}$ and $\mathrm{Ag}-\mathrm{O}$, in order. ${ }^{49}$ All wavelength shifts were represented by dashed vertical lines in Figure S7a. These observations may suggest that metals were bonded to the MCM-41 structure, surface or not, as suggested by the XRD discussions from those catalysts after impregnation with $\mathrm{Cu}, \mathrm{Co}$, and $\mathrm{Ni}$, that evidenced the disappearance of the MCM-41 low angle

\begin{tabular}{|lllll|}
\hline Catalyst & B.E.T. $\left(\mathrm{m}^{2} / \mathrm{g}\right)$ & Pore volume $\left(\mathrm{cm}^{3} / \mathrm{g}\right)$ & $\mathrm{Dp}(\mathrm{nm})$ & Bandgap $(\mathrm{eV})$ \\
\hline $\mathrm{MCM}-41$ & 1275 & 0.631 & 1.98 & 4.37 \\
\hline $\mathrm{Ti} / \mathrm{MCM}-41$ & 871 & 0.570 & 2.62 & 3.18 \\
\hline $\mathrm{Cu}-\mathrm{Ti} / \mathrm{MCM}-41$ & 859 & 0.537 & 2.50 & 2.88 \\
\hline $\mathrm{Co}-\mathrm{Ti} / \mathrm{MCM}-41$ & 832 & 0.490 & 2.35 & 3.11 \\
\hline $\mathrm{Ni}-\mathrm{Ti} / \mathrm{MCM}-41$ & 910 & 0.552 & 2.43 & 3.14 \\
\hline $\mathrm{Ag}-\mathrm{Ti} / \mathrm{MCM}-41$ & 1077 & 0.700 & 2.60 & 3.07 \\
\hline
\end{tabular}

TABLE 1 Textural parameters and bandgap energy values from prepared catalysts 


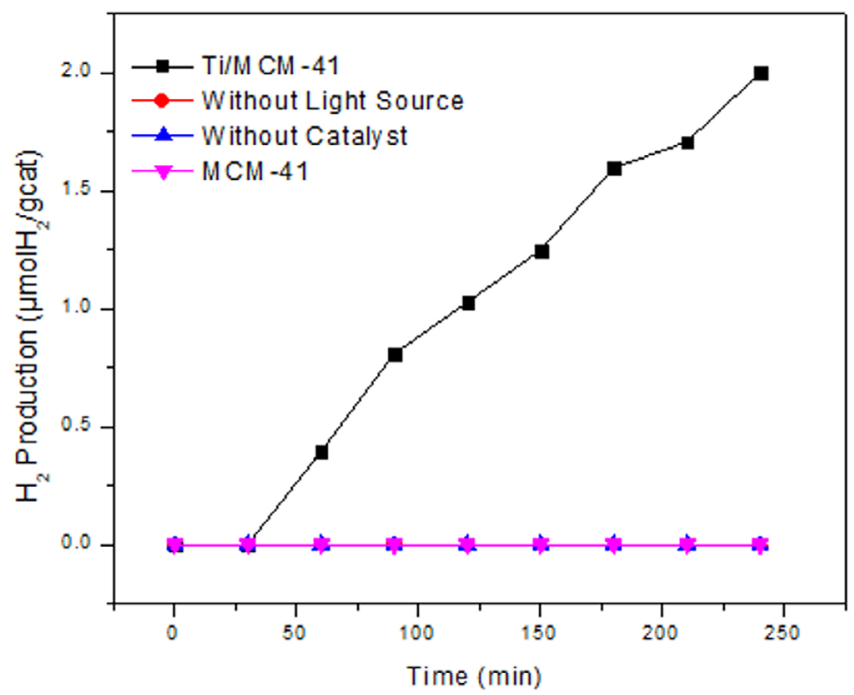

FIGURE 3 Controls tests for $\mathrm{H} 2$ production ( $\mu \mathrm{mol}(\mathrm{H} 2) \mathrm{g}-1)$ realized at an annular reactor./Legend: [catalyst]: $1 \mathrm{gL}^{-1}$; V: $0.6 \mathrm{~L}$ ethanolic solution $10 \%(\mathrm{~m} / \mathrm{m})$; $t$ : $4 \mathrm{~h}$ radiation time and one $18 \mathrm{~W}$ UV-C lamp [Color figure can be viewed at wileyonlinelibrary.com]

planes. Although the low-angle planes of the molecular sieve did not disappear into the catalyst impregnating with $\mathrm{Ag}$, the insertion of this metal in the structure was identified, in addition to the displacements in the IR, also due to the greater porosity in the textural analysis, indicating metallic bonding to the surface.

No peaks in the regions of $720-740$ and $1400-1500 \mathrm{~cm}^{-1}$, corresponding to the aliphatic $\mathrm{C}-\mathrm{H}$ bond vibrations, and in the $2800-3100 \mathrm{~cm}^{-1}$ range, relative to the aliphatic C-H stretch vibrations, indicates the complete CTAB template removal which was used as MCM-41 framework driver. ${ }^{42}$

The addition of metals allowed the bandgap energy reduction (Table 1) for all prepared catalysts when compared to Ti/MCM-41, which shows absorption at a wavelength close to pure titanium. ${ }^{8,19,50}$ The displacement of the length was most evident in the copper impregnated catalyst due to its better charge separation capability (Work Function), or in other words, the copper metal-doped catalyst formed an electron donor level at lower energy. ${ }^{51}$

\section{2 | Cocatalyst investigation}

The hydrogen production tests began with the following control reactions presented in Figure 3: (i) using a molecular sieve (MCM-41) to prove that is not active on its $\mathrm{own}^{4}$; (ii) a Ti/MCM-41 catalyst test to clarify that titanium is the active phase of the material, (iii) a test without a light source to prove that the reaction is photocatalytic, and for lastly (iv) an experiment without a catalyst to prove that the reaction is catalytic and heterogeneous. These reaction test arrangements were chosen to demonstrate that the hydrogen production requires the presence of UV light and photocatalysts. All control experiments were performed using the ethanolic solution as a sacrificial agent, as it is necessary to reduce the photogenerated holes in the photocatalyst

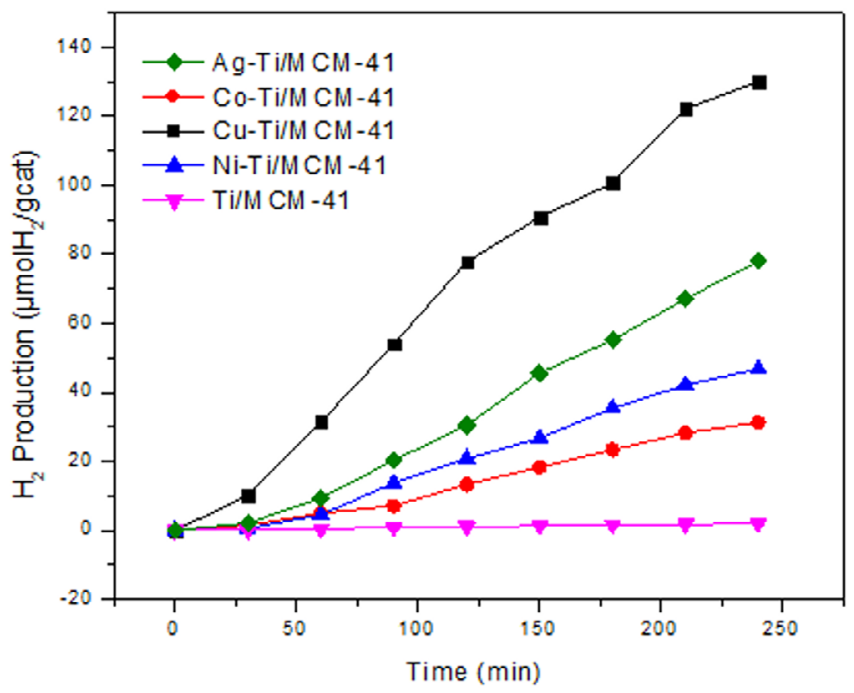

FIGURE $4 \quad$ H2 production by water splitting applying the asprepared photocatalysts./Legend: [catalyst]: $1 \mathrm{gL}^{-1}$; V: $0.6 \mathrm{~L}$ ethanolic solution $10 \%(\mathrm{~m} / \mathrm{m}) ; \mathrm{t}$ : $4 \mathrm{~h}$ radiation time and one $18 \mathrm{~W}$ UV-C lamp [Color figure can be viewed at wileyonlinelibrary.com]

valence band, serving as an antioxidant agent to reduce the electron recombination rate because without this artifice, hydrogen production will never achieve high yields. ${ }^{18,19}$

In compliance with results from $\mathrm{H}_{2}$ generation while applying the metal-doped photocatalysts (Figure 4), it can be seen that the use of ternary photocatalysts markedly increased hydrogen production for any cocatalyst applied. The use of transition metals as a doping agent acted as an electrons trap, which reduced the likelihood of electronic recombination and consequently improved reaction yield. ${ }^{6-8,11,18}$

The best hydrogen production activity was obtained using the $\mathrm{Cu}-\mathrm{Ti} / \mathrm{MCM}-41$ catalyst $\left(130 \mu \mathrm{molH}_{2} \mathrm{~g}^{-1}\right)$. Copper nanoparticles exhibit, within the range of observed values, greater charge separation when compared to the other cocatalysts used, and thus tends to form the Schottky barrier, which encourages the migration of electrons from $\mathrm{TiO}_{2}$ to the cocatalyst, and the holes remain unchanged, thus delaying electronic recombination. ${ }^{19,51}$ In addition, hydrogen production is directly related to the bandgap value, that is, a higher generation of $\mathrm{H}_{2}$ is observed because of the lower bandgap value, which provides greater absorption of the lamp's electromagnetic spectrum (Table 2).

Figure 5 shows the high resolution XPS spectra for the $\mathrm{Cu} 2 \mathrm{p}_{3 / 2}$, O 1 s, Ti $2 p$, and Si $2 p$ regions collected from the samples $1 \% \mathrm{Cu} / 20 \%$ $\mathrm{TiO}_{2} / \mathrm{MCM}-41$ and $20 \% \mathrm{TiO}_{2} / \mathrm{MCM}-41$. As expected, the copper signal was only observed for the sample containing $1 \%$ of $\mathrm{Cu}$, and the chemical components related to $\mathrm{Cu}^{+2}(934.5 \mathrm{eV})$ and $\mathrm{Cu}^{+1}$ $(932.1 \mathrm{eV})^{10,13,52}$ were found at the $\mathrm{Cu} 2 \mathrm{p}_{3 / 2}$ region. Based on the fitting results, one may infer that $\mathrm{Cu}^{+1}$ corresponds to $94 \%$ of the copper species located at the catalyst surface. This is in line with what was observed in the EDS and in the textural analysis, which indicated the presence of copper on the MCM-41 surface. The $\mathrm{Cu}^{+1} / \mathrm{Cu} 0$ reduction potential is higher than $\mathrm{Cu}^{2+} / \mathrm{Cu}^{0}$, indicating that the photoexcited electrons preferentially migrate to $\mathrm{Cu}_{2} \mathrm{O}$ than to $\mathrm{CuO}$, 

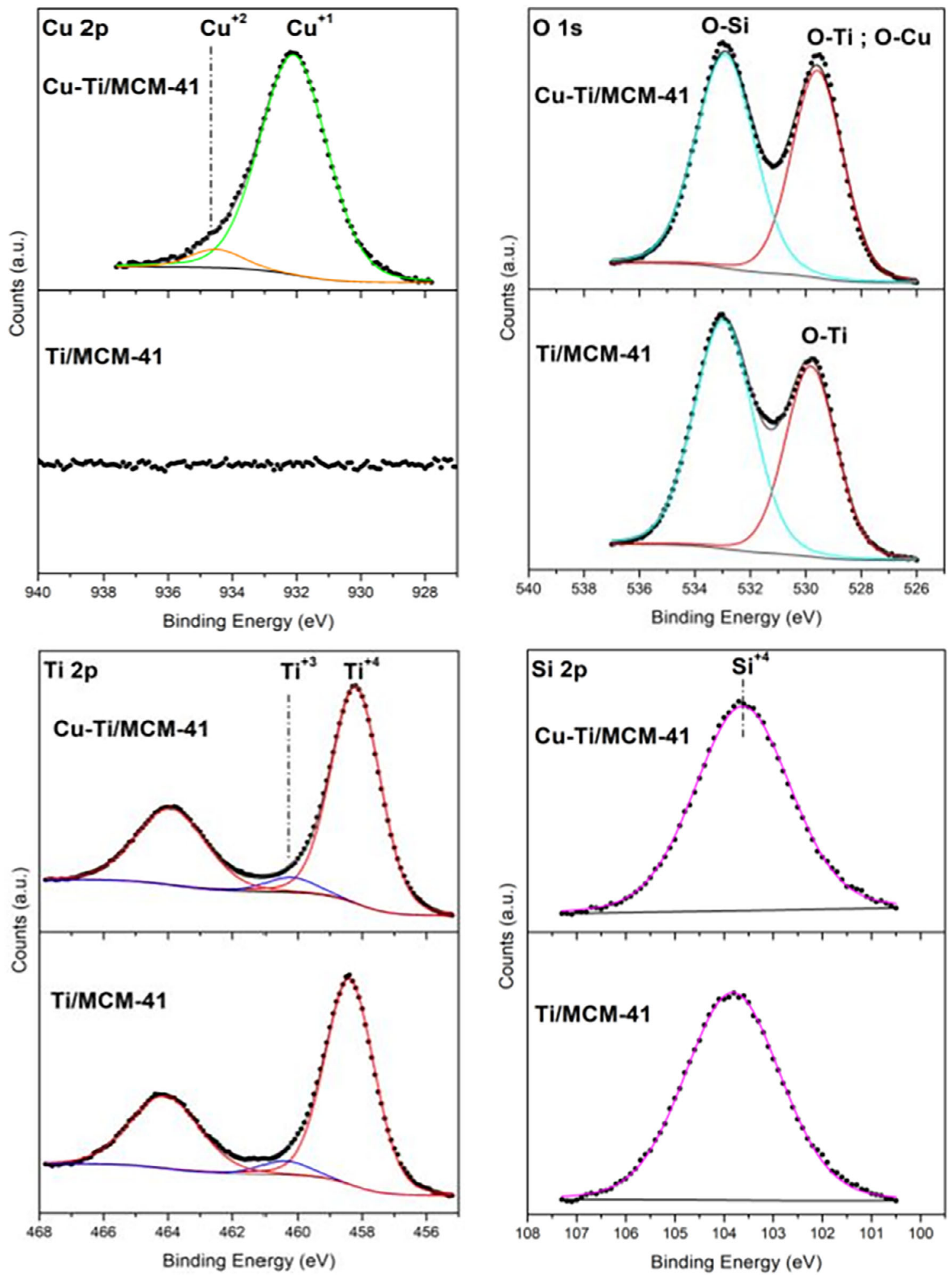

FIGURE 5 XPS spectra for the $\mathrm{Cu} 2 \mathrm{p}_{3 / 2}, \mathrm{O} 1 \mathrm{~s}$, Ti $2 \mathrm{p}$ and $\mathrm{Si} 2 \mathrm{p}$ regions collected from the samples $\mathrm{Cu}-\mathrm{Ti} / \mathrm{MCM}-41$ and Ti/MCM-41 [Color figure can be viewed at wileyonlinelibrary.com]

resulting in better efficiency in charge separation and greater effect on the production of hydrogen for $\mathrm{Cu}^{1+}$ in $\mathrm{Cu}-\mathrm{Ti} / \mathrm{MCM}-41 .{ }^{10,13}$ The O $1 \mathrm{~s}$ region allowed the observation of two distinguishable peaks corresponding to the $\mathrm{O}-\mathrm{Ti}$ and $\mathrm{O}-\mathrm{Si}$ bonds from $\mathrm{TiO}_{2}$ and $\mathrm{SiO}_{2}{ }^{53}$ respectively. It is noteworthy that the presence of copper seems to enhance the O-Ti component, but actually this is due to the contribution of $\mathrm{O}-\mathrm{Cu}$ bonds in the $\mathrm{O} 1 \mathrm{~s}$ spectra, located approximately at the same binding energy as the O-Ti bonds. ${ }^{54}$ In the case of the Ti $2 p$ and 
Si $2 p$ XPS regions, no changes in the oxidation states due to the presence of copper were noticeable, which presents the $\mathrm{Ti}^{+3}, \mathrm{Ti}^{+4}$, and $\mathrm{Si}^{+4}$ chemical components with the same relative intensity for both samples.

In Figure 6, the photoluminescence spectroscopy technique was used to add to the discussion about the best doping metal in the photocatalytic efficiency of $\mathrm{H}_{2}$ generation. In the spectra, the broad bands correspond to the photocatalysts luminescence. The high luminescence of Ti/MCM-41 binary catalyst reflects the difficulty of using catalysts like that in photocatalytic reactions due to the high electronic recombination rate, which increases the catalyst deactivation velocity. ${ }^{21,44}$ However, after metallic impregnation, a reduction in the intensity in the luminescence spectra is noted, indicating that there was a reduction in the electronic recombination rate for all doped metals.

The displacement of photocatalysts spectra after metallic impregnation to longer wavelengths, observed in Figure 6, is justified by the change in maximum-wavelength absorbed by the materials. This change in absorbed wavelengths refers to the value of the bandgap energy that is unique for each material, as indicated by the PAS (Table

TABLE 2 Apparent quantum yield values

\begin{tabular}{llll}
\hline Catalysts & Reactor & Lamps $^{\text {a }}$ & AQY \\
\hline Cu-Ti/MCM-41 & Reactor A & 1 & $1.19 \%$ \\
\hline Co-Ti/MCM-41 & Reactor A & 1 & $0.28 \%$ \\
\hline Ni-Ti/MCM-41 & Reactor A & 1 & $0.43 \%$ \\
\hline Ag-Ti/MCM-41 & Reactor A & 1 & $0.71 \%$ \\
\hline Ti/MCM-41 & Reactor A & 1 & $0.02 \%$ \\
\hline Ti/MCM-41 & Reactor B & 1 & $0.08 \%$ \\
\hline Cu-Ti/MCM-41 & Reactor B & 1 & $1.81 \%$ \\
\hline Cu-Ti/MCM-41 & Reactor B & 2 & $1.81 \%$ \\
\hline Cu-Ti/MCM-41 & Reactor B & 3 & $1.52 \%$ \\
\hline
\end{tabular}

a18 W UV-C lamps varying from 1 to 3 lamps.

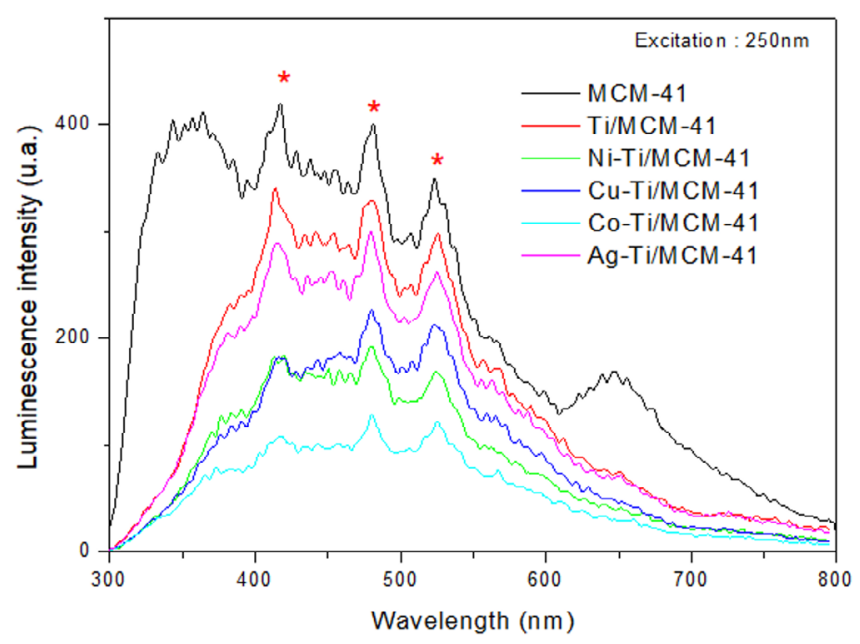

FIGURE 6 Photoluminescence spectra from prepared catalysts [Color figure can be viewed at wileyonlinelibrary.com]
1 and Figure 7); therefore, the materials emit radiation only after this specific wavelength.

The $\mathrm{Cu}$-Ti/MCM-41 catalyst spectrum shows the lowest intensity compared to the others, except when compared to Co-Ti/MCM-41, indicating low energy loss in the electronic recombination process. Otherwise stated, copper has better action on charge sequestration, as expected by the nanoparticle work function, and thus delays electron recombination, allowing it to react with $\mathrm{H}^{+}$ions to form $\mathrm{H}_{2}$, favoring better photocatalytic results.

The reason why the photoluminescence spectrum of the Co-Ti/ MCM-41 photocatalyst was less intense when compared to $\mathrm{Cu}-\mathrm{Ti}$ / MCM-41 is the luminescence resorption in the visible spectrum region, identified by the greater color change presented by the material, leaving it darker and reducing the intensity of the spectrum. ${ }^{8}$ The photoacoustic spectra shown in Figure 7 indicate that the metaldoped photocatalysts exhibit absorption in the visible region, between 400 and $800 \mathrm{~nm}$ (dotted demarcated region), unlike MCM-41 and $\mathrm{Ti} / \mathrm{MCM}-41$ binary catalyst that have almost no absorption in the same region. ${ }^{44}$ And this absorption in the visible region, found in photocatalysts, is the reason for the reabsorption of photoluminescence in the same region, mainly in the spectrum of Co-Ti/ MCM-41, causing its low intensity.

The unequal behavior at the beginning of the photoluminescence spectrum between the steeper curve MCM-41 and the smooth curve of doped catalysts (Figure 6) is due to the "high pass" optical filter employed $(\lambda=320 \mathrm{~nm}$ ). The filter is necessary to avoid the second harmonic of the excitation wavelength $(\lambda=250 \mathrm{~nm})$ at $500 \mathrm{~nm}$. This filter becomes useful because if it had not been used in the second harmonic the intensity would go high enough to overlap the entire spectrum of materials. The MCM-41 peak located at $660 \mathrm{~nm}$ represents the second harmonic of the $330 \mathrm{~nm}$ peak of the same sample, the same behavior is observed for the other materials, but to a lesser extent due to the low signal. The peaks marked with asterisks in all spectra are sinuosities from the system (416, 481, and $522 \mathrm{~nm}$ ), and are inherent of the equipment itself, which is evidenced by the non-appearance of their second harmonics at 832, 962, and $1044 \mathrm{~nm}$.

Thus, the effects of the increased absorption spectrum (lower bandgap), higher trapping electrons capability (greater work function), good surface metal cocatalyst distribution (reported by EDS discussion in the Supplementary Material) and major presence of the most active oxidation state of copper, $\mathrm{Cu}^{1+}$, on catalyst surface (reported by XPS discussions). All those observations delay the electrons recombination rate from the catalyst, leaving them free on the surface to promote redox reactions of water photolysis, ratifying the $\mathrm{Cu}-\mathrm{Ti} /$ MCMC-41 as the most photoactive catalyst in hydrogen generation via water splitting studied in this article.

\section{3 | Reactors comparison}

In order to compare the geometry and reaction system of annular (Reactor A) and quartz (Reactor B) reactors, the same catalyst ( $\mathrm{Cu}-\mathrm{Ti}$ / 


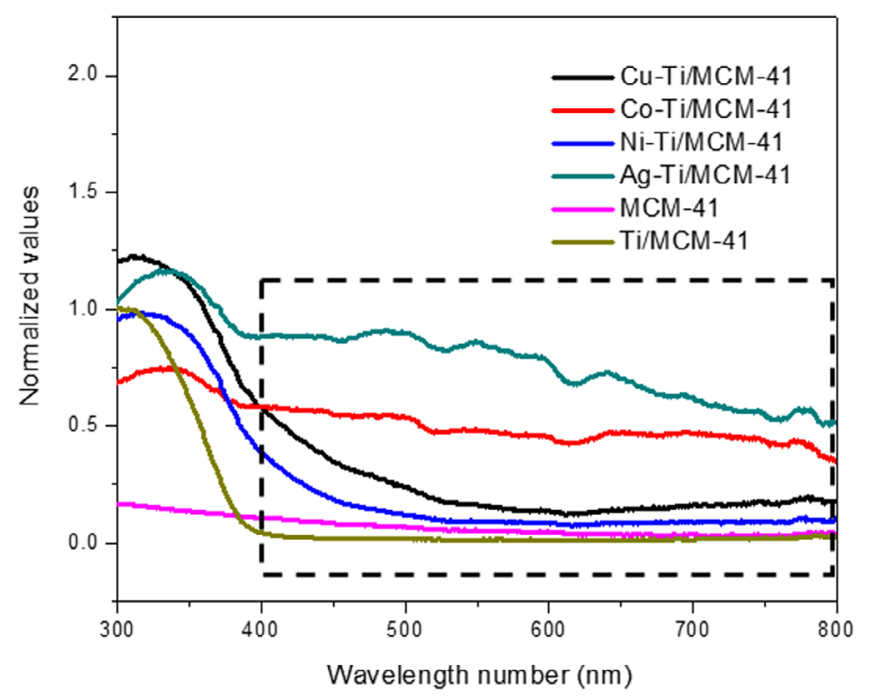

FIGURE 7 Photoacoustic spectroscopy for MCM-41 and prepared photocatalysts [Color figure can be viewed at wileyonlinelibrary.com]

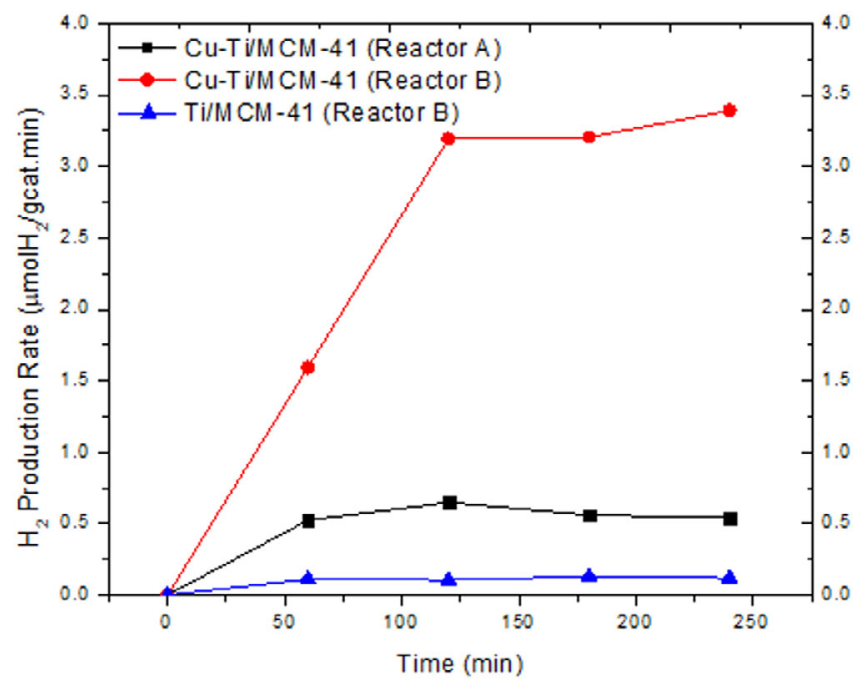

FIGURE 8 Comparison of $\mathrm{H} 2$ generation rate by photocatalytic water splitting between annular (Reactor $A$ ) and quartz (Reactor $B$ ) reactors./Legend: [catalyst]: $1 \mathrm{gL}^{-1}$; $\mathrm{V}: 0.6 \mathrm{~L}$ (Reactor $\mathrm{A}$ ) and $30 \mathrm{ml}$ (Reactor B) of $10 \%(w / w)$ ethanolic solution; $t$ : $4 \mathrm{~h}$ of irradiation time and irradiated by one $18 \mathrm{~W}$ UV-C lamp [Color figure can be viewed at wileyonlinelibrary.com]

MCM-41) was employed in a test under the same temperature conditions $\left(25^{\circ} \mathrm{C}\right)$, pressure (atmospheric), radiation (one $18 \mathrm{~W}$ UV-C lamp), catalyst concentration $\left(1 \mathrm{gL}^{-1}\right.$ ) and sacrificial agent (ethanolic solution, $10 \% \mathrm{v} / \mathrm{v})$. In Figure 8, the reaction rates of $\mathrm{H}_{2}$ production were allocated to the different reactors.

In conformity with Figure 8 , it is seen that the quartz reactor (Reactor B) has a hydrogen generation rate approximately seven times higher than the annular reactor (Reactor A) operated under the same experimental conditions. The four main observations that justify the superiority or advantage of using the quartz reactor over the annular are:

1. The first operational difference observed between the systems is the perfect homogenization of reaction system $A$ throughout the time interval guaranteed by a magnetic stirrer. The fluid movement plays an important role in a reaction system, as it provides a homogeneous distribution of reagents, pollutants, catalysts, temperature and bubbles within the reactor, but it does not guarantee the photoreactor isoactinicity, that is, the uniform light distribution in the reactor volume. ${ }^{36,39,55}$ The absence of agitators (reaction system B) may be an advantage as it makes the cost less expensive and facilitates the scale-up of photoreactor designs like quartz ones with an external radiation source attached to a refractory panel. Scaling-up photoreactors with an internal radiation source and large agitators may require a lot of maintenance, hazardous care, as well as elevated operating expenses such as baffle insertion to avoid vortex formation, and also height increment of the photoreactor to avoid dark areas with a diameter enlargement. ${ }^{35}$

2. Reactor B has a smaller volume compared to Reactor A, approximately $90 \%$ smaller, and has a $1.3 \mathrm{~cm}$ thin layer of water, comprising a light exposure area of approximately $96 \mathrm{~cm}^{2}$. Reactor $A$ has a light exposure area of $101 \mathrm{~cm}^{2}$, referring to the external area of the lamp's protective tube in contact with the solution, which is at a distance of $3 \mathrm{~cm}$ from the inner wall of the reactor. Even though the area of light exposure is similar, the light distribution within the photoreactors is the main reason why Reactor B obtained the highest yield in hydrogen production. The higher ratio of light exposure area to liquid phase volume $(A / V)$ is the reason for the best light distribution in reactor $\mathrm{B}$, which indicates that the photons focus on $3.2 \mathrm{~cm}^{2}$ per volume unit compared to $0.16 \mathrm{~cm}^{2}$ of Reactor A. Greater A/V ratio values also indicate that dark zones are less likely to form inside the reactors. Photon blocking is the major factor that causes the dark area inside the reactors. The light block is caused by photocatalysts photons absorption, which subsequently decreases the photocatalytic activity by not activating them at that instant. The larger the volume to be penetrated by light, the more likely it is that all photons will be absorbed before they impact the inner wall of the reactor. This reasoning can be easily understood by inverting the values of $A / V$ to $V / A$, resulting in 0.3 and $6.3 \mathrm{ml} \mathrm{cm}^{-2}$ for reactors $B$ and $A$, respectively. The nonformation of dark zones in reactor $B$ is due to the fact that the photoreactor is covered by a refractory material that allows light to penetrate the entire volume of the thin layer of water on all sides. This quartz tube reactor configuration is very close to the quasi-isoactinicity presented by Brucato et al. ${ }^{55}$

3. The quartz reactor allows the application of a higher catalyst concentration due to the higher exposure of light area per unit volume, making it a great economic advantage. The concentration of $1 \mathrm{~g} / \mathrm{L}$ for annular reactor ( $10 \mathrm{~cm}$ inner diameter), can be considered very high and it is very likely that dark regions were formed. It was reported in the literature that LVREA profiles of annular reactors 


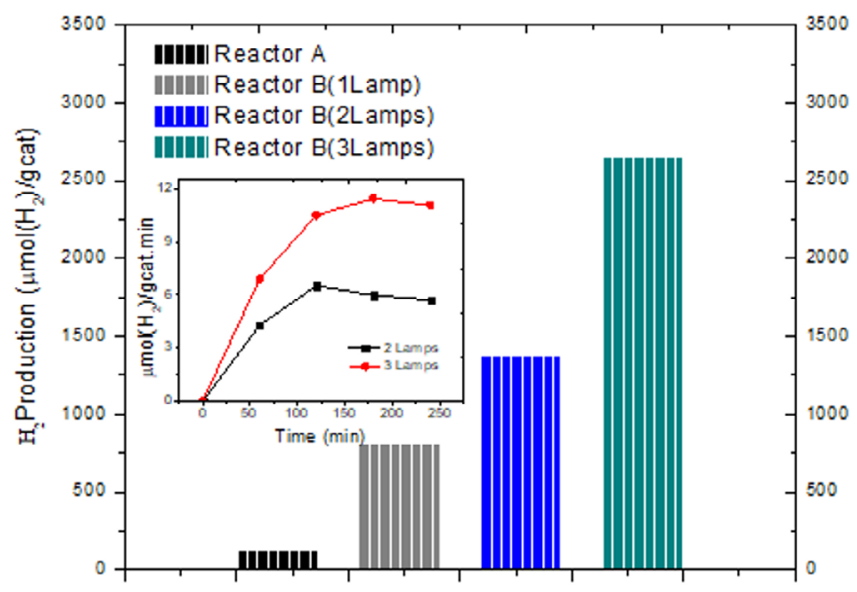

FIGURE 9 Evaluation of the maximum $\mathrm{H} 2$ generation from quartz reactor (Reactor $\mathrm{B}$ ) according to the number of lamps used. Internal chart: $\mathrm{H} 2$ production rate./Legend: [catalyst]: $1 \mathrm{gL}^{-1} ; \mathrm{V}: 0.6 \mathrm{~L}$ (Reactor A) and $30 \mathrm{ml}$ (Reactor B) of $10 \%(\mathrm{w} / \mathrm{w})$ ethanolic solution; t: $4 \mathrm{~h}$ of irradiation time and irradiated by one 18 W UV-C lamp [Color figure can be viewed at wileyonlinelibrary.com]

with smaller inner diameters like, $0.85,2.6,3.52$, and $6.5 \mathrm{~cm}$, obtained optimal $\mathrm{TiO}_{2}$ concentrations (1.0, 0.25, 0.14, and $0.4 \mathrm{~g} / \mathrm{L}$, respectively) lower than that used in the present study. ${ }^{34,36-38}$

4. Another way to evaluate the differences in $\mathrm{H}_{2}$ production of the two photoreactors is by the apparent quantum yield (AQY) described in the Supplementary Material. AQY analysis comprehends the whole photocatalytic system, including the reactor geometry and the radiation source information. The non-utilization of all the photons emitted by the lamp (Figure S7b in the Supplementary Material) is one of the reasons that a low quantum yield was obtained (Table 2), besides light scattering. Photocatalysts only absorb wavelengths less than or equal to the maximum wavelength, relative to the bandgap energy value (Table 1), so longer wavelengths are not used for electronic excitation (Figure S7b). In agreement with Figure S7b, Cu-Ti/MCM-41 catalyst absorbs more wavelengths from the lamp spectrum than other catalysts. The higher quantum efficiency calculated for reactor $B(1.81 \%)$ compared to reactor $A(1.19 \%)$ is a strong indication that it is advantageous to design reactors with higher $A / V$ ratios, not just larger light exposure areas, as observed experimentally. Thus, the findings complement Brucato's and coauthors' conclusions when they described the shape of a quasi-isoactinic photoreactor, which is a semi-infinite reactor with two plates separated by a short distance and radiated on both sides. ${ }^{55}$

When the full power of the arrangement of the lamps at Reactor $B$ is in use, hydrogen generation is even more prominent (Figure 9). With two lamps, a production of $1372.2 \mu \mathrm{mol}\left(\mathrm{H}_{2}\right) \mathrm{g}_{\mathrm{cat}}{ }^{-1}$ was obtained in $4 \mathrm{~h}$ of reaction, 1.7 times higher than the same reaction made with only one lamp. Likewise, the reaction when performed with 3 lamps (maximum luminous power) produced $2651.5 \mu \mathrm{mol}\left(\mathrm{H}_{2}\right) \mathrm{g}_{\mathrm{cat}}{ }^{-1}, 1.9$ and 3.3 times higher than with 2 and 1 lamps, respectively, the same which was observed by Bednarczyh and coauthors, who reported that with an increase light intensity there was an increase in hydrogen production. ${ }^{19}$

Unlike hydrogen production, the AQY did not increase with the increase in the number of lamps used (Table 2). With the increase in the number of lamps used there is an increase in the amount of photons emitted, on the other hand, the amount of catalyst remains the same, that is, the whole active area for photon absorption has already been irradiated and part of the emitted photons are not used for the electronic excitation. It is noteworthy that the increase in catalyst mass employed in the photocatalytic reaction is not a guarantee of better yield in $\mathrm{H}_{2}$ production since there is an optimum concentration after which a formation of dark zones occurs, as discussed in the light distribution inside the reactors.

The hydrogen production rates obtained in the quartz reactor varied according to the set of lamps used (1, 2, and 3 lamps) (Figure 9) are, in other units, 203.4, 360, and $686.4 \mu \mathrm{mol}\left(\mathrm{H}_{2}\right) \mathrm{g}^{-1} \mathrm{~h}^{-1}$, respectively, and are higher than the average hydrogen production found in literature for quartz reactors. ${ }^{18}$ The main differences between the reaction systems found in literature and this present work can be highlighted as:

1. Most methods use $300 \mathrm{~W}$ Xe lamps, a much higher wattage than the ones used in the present study (maximum $54 \mathrm{~W}$ corresponding to the use of three lamps of $18 \mathrm{~W}$ lamps each $)^{8,17}$;

2. Many methods have used noble metals in the composition of catalysts, making the process more costly $6,9,16$;

3. Most of the cases applied sacrificial agents with more $\alpha-\mathrm{H}$ atoms adjacent to the hydroxyl groups, camouflaging hydrogen production via water splitting to alcohols or mineralizing agents, which are considered more toxic and are employed in higher concentrations when compared to the $10 \%$ ethanolic solution used in the present method.

\section{CONCLUSION}

The $\mathrm{Cu}-\mathrm{Ti} / \mathrm{MCM}-41$ photocatalyst proved to be the most effective in generating hydrogen from photocatalytic water splitting, with a production of $130 \mu \mathrm{molH}_{2} \mathrm{~g}^{-1}$ and a quantum efficiency of $1.19 \%$ in $4 \mathrm{~h}$ of irradiation. The lower bandgap energy value and the lower photoluminescence spectrum response indicated that the photocatalyst is excited by most of the lamp emission spectrum and has the lowest electronic recombination rate, respectively. The lower blockade of the photons emitted by the lamp provided better results for the quartz reactor, with a production of $814.2 \mu \mathrm{molH}_{2} \mathrm{~g}^{-1}$ and a quantum efficiency of $1.81 \%$. Counterintuitively, a larger area of light exposure does not guarantee higher yields under the same experimental conditions, as the areas are close between the reactors. The higher $A / V$ of the quartz reactor indicates that in a volume unit, an area of $3.2 \mathrm{~cm}^{2}$ is irradiated, against $0.16 \mathrm{~cm}^{2}$ of the annular reactor, having a better utilization of the emitted photons, fact confirmed by the AQY, which introduces effects of the geometry and light source information in the calculations. In this way, the quartz reactor allows the application of higher catalyst concentrations and simplifies the industrial scale-up design. 


\section{ACKNOWLEDGMENTS}

The authors would like to thank the Coordenação de Aperfeiçoamento de Pessoal de Nível Superior (CAPES) and Conselho Nacional de Desenvolvimento Científico e Tecnológico (CNPq 406750/2016-5) for the financial support and to the Complexo de Centrais de Apoio à Pesquisa (COMCAP) for the availability of material characterization equipment. The authors would like to thank Laboratory of Adsorption and Ion Exchange for realization of $\mathrm{N}_{2}$ physisorption analysis.

\section{DATA AVAILABILITY STATEMENT}

The data that support the findings of this study are available from the corresponding author upon reasonable request.

\section{ORCID}

Jean César Marinozi Vicentini (D) https://orcid.org/0000-0003-1648476X

Gimerson Weigert Subtil (D) https://orcid.org/0000-0002-5695-2321 Fernanda de Oliveira Tavares (D) https://orcid.org/0000-0002-37131413

Jonder Morais (D) https://orcid.org/0000-0002-4143-1208

\section{REFERENCES}

1. Abrokwah RY, Deshmane VG, Kuila D. Comparative performance of M-MCM-41 (M: Cu, Co, Ni, Pd, $\mathrm{Zn}$ and $\mathrm{Sn}$ ) catalysts for steam reforming of methanol. J Mol Catal A Chem. 2016;425:10-20.

2. Deshmane VG, Owen SL, Abrokwah RY, Kuila D. Mesoporous nanocrystalline $\mathrm{TiO}_{2}$ supported metal (cu, co, Ni, $\mathrm{Pd}, \mathrm{Zn}$, and $\mathrm{Sn}$ ) catalysts: effect of metal-support interactions on steam reforming of methanol. J Mol Catal A Chem. 2015;408:202-213.

3. Cakiryilmaz N, Arbag H, Oktar N, Dogu G, Dogu T. Catalytic performances of $\mathrm{Ni}$ and $\mathrm{cu}$ impregnated MCM-41 and $\mathrm{Zr}-\mathrm{MCM}-41$ for hydrogen production through steam reforming of acetic acid. Catal Today. 2019;323(June 2018):191-199.

4. Nejat T, Jalalinezhad P, Hormozi F, Bahrami Z. Hydrogen production from steam reforming of ethanol over $\mathrm{Ni}$-co bimetallic catalysts and MCM-41 as support. J Taiwan Inst Chem Eng. 2019;97:216-226.

5. Shu H, Lu L, Zhu S, et al. Ultra small cobalt nanoparticles supported on MCM41: one-pot synthesis and catalytic hydrogen production from alkaline borohydride. Catal Commun. 2019;118(July 2018):30-34.

6. Fernandes NRC, Olsen Scaliante MHN, Yassue-Cordeiro PH, Schwaab M, Barbosa-Coutinho E, Enzweiler $\mathrm{H}$. Evaluation of Pd$\mathrm{TiO}_{2} / \mathrm{ZSM}-5$ catalysts composition effects on hydrogen production by photocatalytic water splitting. Int J Hydrog Energy. 2018;43(13): 6515-6525.

7. Liao C, Li J, Zhang Y, et al. Visible light driven photocatalytic $\mathrm{H}_{2}$ generation property of trigonal $\mathrm{ZnIn}_{2} \mathrm{~S}_{4}$ prepared by high temperature solid state reaction. Mater Lett. 2019;248:52-54.

8. Chen W, Liu M, Wang Y, Gao L, Dang H, Mao L. Non-noble metal co as active sites on $\mathrm{TiO}_{2}$ nanorod for promoting photocatalytic $\mathrm{H}_{2}$ production. Mater Res Bull. 2019;116(December 2018):16-21.

9. Nguyen NT, Ozkan S, Hejazi S, et al. Providing significantly enhanced photocatalytic $\mathrm{H}_{2}$ generation using porous PtPdAg alloy nanoparticles on spaced $\mathrm{TiO}_{2}$ nanotubes. Int J Hydrog Energy. 2019;44(41):2296222971.

10. Liu $\mathrm{Y}$, Ren $\mathrm{H}$, Lv H, Gong Z, Cao Y. An insight into the trifunctional roles of $\mathrm{Cu}_{2}(\mathrm{OH})_{2} \mathrm{CO}_{3}$ cocatalyst in boosting the photocatalytic $\mathrm{H}_{2}$ evolution activity over $\mathrm{Zn}_{0.5} \mathrm{Cd}_{0.5} \mathrm{~S}$ nanoparticles. Appl Surf Sci. 2019; 484(January):1061-1069.
11. Banerjee $T$, Mukherjee $A$. Overall water splitting under visible light irradiation using nanoparticulate $\mathrm{RuO}_{2}$ loaded $\mathrm{Cu}_{2} \mathrm{O}$ powder as photocatalyst. Energy Procedia. 2014;54:221-227.

12. Jing D, Guo L, Zhao L, et al. Efficient solar hydrogen production by photocatalytic water splitting: from fundamental study to pilot demonstration. Int J Hydrog Energy. 2010;35(13):7087-7097.

13. Xu S, Ng J, Zhang X, Bai H, Sun DD. Fabrication and comparison of highly efficient cu incorporated $\mathrm{TiO}_{2}$ photocatalyst for hydrogen generation from water. Int J Hydrog Energy. 2010;35(11):5254-5261.

14. Nong G, Li M, Chen Y, Zhou Z, Wang S. Simulation of energy conversion in a plant of photocatalysts water splitting for hydrogen fuel. Energy. 2015;81:471-476.

15. Kudo A, Miseki Y. Heterogeneous photocatalyst materials for water splitting. Chem Soc Rev. 2009;38(1):253-278.

16. Qian K, Huang W. A new strategy to enhance quantum efficiency of photo-mediated hydrogen evolution. Catal Today. 2019;327:334-339.

17. Liu Z, Wang J, Ma H, et al. A new natural layered clay mineral applicable to photocatalytic hydrogen production and/or degradation of dye pollutant. Environ Prog Sustain Energy. 2018;37(3):1003-1010.

18. Fajrina N, Tahir M. A critical review in strategies to improve photocatalytic water splitting towards hydrogen production. Int J Hydrog Energy. 2019;44(2):540-577.

19. Bednarczyk K, Stelmachowski M, Gmurek M. The influence of process parameters on photocatalytic hydrogen production. Environ Prog Sustain Energy. 2019;38(2):680-687.

20. Ghosh P, Chelli VR, Giri AS, Golder AK. Steroid glycosides as potential analytes for cu-doping on $\mathrm{TiO}_{2}$ for photocatalytic water treatment. Environ Prog Sustain Energy. 2018;37(6):1973-1981.

21. Jia W, Liu T, Li Q, Yang J. Highly efficient photocatalytic reduction of $\mathrm{CO}_{2}$ on surface-modified Ti-MCM-41 zeolite. Catal Today. 2019;335 (November 2018):221-227.

22. Matsuoka $M$, Kitano $M$, Takeuchi $M$, Tsujimaru $K$, Anpo $M$, Thomas JM. Photocatalysis for new energy production. Catal Today. 2007;122(1-2):51-61.

23. Sreethawong $\mathrm{T}$, Yoshikawa $\mathrm{S}$. Comparative investigation on photocatalytic hydrogen evolution over $\mathrm{Cu}^{-}, \mathrm{Pd}-$, and au-loaded mesoporous $\mathrm{TiO}_{2}$ photocatalysts. Catal Commun. 2005;6(10):661-668.

24. Sreethawong T, Puangpetch T, Chavadej S, Yoshikawa S. Quantifying influence of operational parameters on photocatalytic $\mathrm{H}_{2}$ evolution over Pt-loaded nanocrystalline mesoporous $\mathrm{TiO}_{2}$ prepared by singlestep sol-gel process with surfactant template. J Power Sources. 2007; 165(2):861-869.

25. Chen W, Chan A, Sun-waterhouse D, Llorca J, Idriss $H$, Waterhouse GIN. Performance comparison of $\mathrm{Ni} / \mathrm{TiO}_{2}$ and $\mathrm{Au} / \mathrm{TiO}_{2}$ photocatalysts for $\mathrm{H}_{2}$ production in different alcohol-water mixtures. J Catalys. 2018;367:27-42.

26. Saravanan R, Manoj D, Qin J, et al. Mechanothermal synthesis of ag/$\mathrm{TiO}_{2}$ for photocatalytic methyl orange degradation and hydrogen production. Process Saf Environ Prot. 2018;120:339-347.

27. Vicentini JCM, Medeiros GB, Watanabe MCC, Piccoli KR, Camilo FC, Scaliante MHNO. Synergistic effect between oxides semiconductors and zeolites on photobleaching of RB250 textile dye. Acta Sci Technol. 2018;40:1-8.

28. Saad EM, Hassan HMA, Soltan MS, Butler IS, Mostafa SI. Removal of copper(II) ions from aqueous media by chemically modified MCM-41 with $\mathrm{N}$-(3-[trimethoxysilyl]propyl)ethylenediamine and its 4-hydroxysalicylidene Schiff-base. Environ Prog Sustain Energy. 2018;37(2):746-760.

29. Shu $Y$, Shao $Y$, Wei $X$, et al. Synthesis and characterization of NiMCM-41 for methyl blue adsorption. Microporous Mesoporous Mater. 2015;214:88-94.

30. Parida K, Mishra KG, Dash SK. Adsorption of toxic metal ion $\mathrm{Cr}(\mathrm{VI})$ from aqueous state by $\mathrm{TiO}_{2}-\mathrm{MCM}-41$ : equilibrium and kinetic studies. J Hazard Mater. 2012;241-242:395-403.

31. Deshmane VG, Abrokwah RY, Kuila D. Synthesis of stable cu-MCM41 nanocatalysts for $\mathrm{H}_{2}$ production with high selectivity via steam 
reforming of methanol. Int J Hydrog Energy. 2015;40(33):1043910452.

32. Bernal C, Mesa M, Jaber M, Guth JL, Sierra L. Contribution to the understanding of the formation mechanism of bimodal mesoporous MCM41-type silica with large defect cavities. Microporous Mesoporous Mater. 2012;153:217-226.

33. Ngo TA, Kim J, Kim SK, Kim SS. Pyrolysis of soybean oil with $\mathrm{H}$ ZSM5 (proton-exchange of zeolite Socony Mobil \#5) and MCM41 (Mobil composition of matter no. 41) catalysts in a fixed-bed reactor. Energy. 2010;35(6):2723-2728.

34. Moreira J, Serrano B, Ortiz A, De Lasa H. Evaluation of photon absorption in an aqueous $\mathrm{TiO}_{2}$ slurry reactor using Monte Carlo simulations and macroscopic balance. Ind Eng Chem Res. 2010;49(21): 10524-10534.

35. Valadés-Pelayo PJ, Guayaquil Sosa F, Serrano B, de Lasa H. Eightlamp externally irradiated bench-scale photocatalytic reactor: scale-up and performance prediction. Chem Eng J. 2015;282: 142-151.

36. Akach J, Ochieng A. Monte Carlo simulation of the light distribution in an annular slurry bubble column photocatalytic reactor. Chem Eng Res Des. 2018;129:248-258.

37. Casado C, García-Gil Á, van Grieken R, Marugán J. Critical role of the light spectrum on the simulation of solar photocatalytic reactors. Appl Catal B Environ. 2019;252(October 2018):1-9.

38. Zekri M e M, Colbeau-Justin C. A mathematical model to describe the photocatalytic reality: what is the probability that a photon does its job? Chem Eng J. 2013;225:547-557.

39. Camera-Roda G, Augugliaro V, Cardillo AG, et al. A reaction engineering approach to kinetic analysis of photocatalytic reactions in slurry systems. Catal Today. 2016;259:87-96.

40. Kočí K, Reli M, Kozák O, et al. Influence of reactor geometry on the yield of $\mathrm{CO}_{2}$ photocatalytic reduction. Catal Today. 2011;176(1): 212-214.

41. Kecht J, Schlossbauer A, Bein T. Selective functionalization of the outer and inner surfaces in mesoporous silica nanoparticles. Chem Mater. 2008;20(23):7207-7214.

42. Chaliha S, Bhattacharyya KG. Fe(III)-, co(II)- and Ni(II)-impregnated MCM41 for wet oxidative destruction of 2,4-dichlorophenol in water. Catal Today. 2009;141(1-2):225-233.

43. Pirouzmand $M$, Mahdavi Anakhatoon $M$. $\beta$-Cyclodextrin containing co/MCM-41 as a catalyst for the production of biodiesel from waste cooking oil. Environ Prog Sustain Energy. 2018;37(5):1770-1773.

44. Chen D, Yang Y, Zhang X, Wang X, Xu Y, Qian G. Mesoporous composite $\mathrm{NiCr}_{2} \mathrm{O}_{4} / \mathrm{Al}-\mathrm{MCM}-41$ : a novel photocatalyst for enhanced hydrogen production. Int J Hydrog Energy. 2019;44(33):18123-18133.

45. Moreno-Recio M, Santamaría-González J, Maireles-Torres P. Brönsted and Lewis acid ZSM-5 zeolites for the catalytic dehydration of glucose into 5-hydroxymethylfurfural. Chem Eng J. 2016;303:22-30.
46. Hao K, Shen B, Wang Y, Ren J. Influence of combined alkaline treatment and Fe-Ti-loading modification on ZSM-5 zeolite and its catalytic performance in light olefin production. J Ind Eng Chem. 2012;18(5):1736-1740.

47. Chen D, Qu Z, Zhang W, Li X, Zhao Q, Shi Y. TPD and TPSR studies of formaldehyde adsorption and surface reaction activity over ag/MCM-41 catalysts. Colloids Surfaces A Physicochem Eng Asp. 2011; 379(1-3):136-142.

48. Sun $\mathrm{H}$, Chen J, Liu S, et al. Photocatalytic $\mathrm{H}_{2}$ evolution of porous silicon derived from magnesiothermic reduction of mesoporous $\mathrm{SiO}_{2}$. Int J Hydrog Energy. 2019;44(14):7216-7221.

49. Malika M, Rao CV, Das RK, Giri AS, Golder AK. Evaluation of bimetal doped $\mathrm{TiO}_{2}$ in dye fragmentation and its comparison to mono-metal doped and bare catalysts. Appl Surf Sci. 2016;368(3):316-324.

50. Singhal N, Ali A, Vorontsov A, Pendem C, Kumar U. Efficient approach for simultaneous $\mathrm{CO}$ and $\mathrm{H}_{2}$ production via photoreduction of $\mathrm{CO}_{2}$ with water over copper nanoparticles loaded $\mathrm{TiO}_{2}$. Appl Catal A Gen. 2016;523:107-117.

51. Low J, Cheng B, Yu J. Surface modification and enhanced photocatalytic $\mathrm{CO}_{2}$ reduction performance of $\mathrm{TiO}_{2}$ : a review. Appl Surf Sci. 2017;392:658-686.

52. Moulder JF, Stickle WF, Sobol PE, Bomben KD. Handbook of X-Ray Photoelectron Spectroscopy. Eden Prairie, Minnesota: Perkin-Elmer Corporation Physical Electronics Division; 1992.

53. Bharti B, Kumar S, Lee HN, Kumar R. Formation of oxygen vacancies and $\mathrm{Ti}^{3+}$ state in $\mathrm{TiO}_{2}$ thin film and enhanced optical properties by air plasma treatment. Sci Rep. 2016;6(May):1-12.

54. Han J, Chang J, Wei R, et al. Mechanistic investigation on tuning the conductivity type of cuprous oxide $\left(\mathrm{Cu}_{2} \mathrm{O}\right)$ thin films via deposition potential. Int J Hydrog Energy. 2018;43(30):13764-13777.

55. Brucato A, Grisafi F, Rizzuti L, Sclafani A, Vella G. Quasi-isoactinic reactor for photocatalytic kinetics studies. Ind Eng Chem Res. 2007;46 (23):7684-7690.

\section{SUPPORTING INFORMATION}

Additional supporting information may be found online in the Supporting Information section at the end of this article.

How to cite this article: Vicentini JCM, Manieri R, Subtil GW, et al. Photocatalytic water splitting with noble-metal free cocatalysts for a comprehensive study of two nonidentical photoreactors designs. Environ Prog Sustainable Energy. 2021; 40:e13557. https://doi.org/10.1002/ep.13557 